\title{
Exploring the protective effect of Modified Xiaochaihu Decoction against hepatic steatosis and inflammation by network pharmacology and validation in ageing rats
}

Siting Gao ${ }^{1,2}$, Tongzhuang Wang ${ }^{1,2}$, XueKuan Huang ${ }^{1,2}$, Yaqian Jin ${ }^{1,3}, Y u X u^{1,2}, Y u m e n g ~ X i{ }^{1,2}$, Jun Zhang ${ }^{1,3}$, Yan Luo ${ }^{1,2}$, Hongling $X u^{1,2}$, Hongli Guo ${ }^{1,2}$, Dazhi Ke ${ }^{4}$ and Jianwei Wang ${ }^{1,2^{*}}$ (1)

\begin{abstract}
Background: Based on therapy with syndrome differentiation and clinical studies on Xiaochaihu decoction (XCHD), we hypothesize that Modified Xiaochaihu Decoction (MXD) has an ability to ameliorate non-alcoholic fatty liver disease (NAFLD). This study aims to elucidate the pharmacological efficacy of MXD and its mechanism in the treatment of NAFLD by network pharmacology and experimental validation.

Methods: The active ingredients in MXD and their potential targets were identified using network analysis followed by experimental validation. First, we used data on the ingredients and targets obtained from professional database and related literature to do PPI network analysis, GO functional analysis, and KEGG pathway enrichment analysis. Core targets identified by network pharmacology were then tested in natural ageing female rats model. Indexes of lipid and glucose homeostasis were determined enzymatically and/or histologically. Gene expression was analyzed by realtime PCR and/or Western blot (WB).

Results: In total, 4009 NAFLD-related targets and 1953 chemical ingredients of MXD were obtained. In-depth network analysis of 140 common targets indicated that MXD played a critical role in anti-NAFLD via multiple targets and pathways. Based on the data of PPI analysis, GO functional enrichment analysis, KEGG pathway enrichment analysis, and literatures on the mechanism of NAFLD, we chose the core targets related to lipid metabolism (SREBP-1C, ChREBP, FASN, PPARa, and ACACA) and inflammation (IL-6 and NF-KB) to do further study. Significantly, in further animal verification experiment we using naturally ageing rats with NAFLD as a model, we found that MXD administration ameliorated age-related NAFLD and mechanistically down-regulated the mRNA/protein expression of core targets in lipid metabolism and inflammation related pathways such as FASN, ACACA, IL-6, and NF-KB. In addition, 12 of 24 potential ingredients acting on verified targets came from $\mathrm{BC}$, and 11 of 24 potential ingredients acting on verified targets were derived from SM, implying that both BC and SM served as the key role in MXD against NAFLD.
\end{abstract}

\footnotetext{
*Correspondence: wangjianwei@cqmu.edu.cn; wjwcq68@163.com

${ }^{1}$ Chongqing Key Laboratory of Traditional Chinese Medicine

for Prevention and Cure of Metabolic Diseases, Chongqing Medical

University, Chongqing, China

Full list of author information is available at the end of the article
}

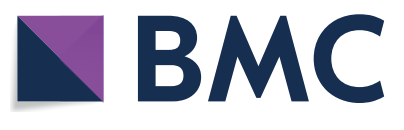

(c) The Author(s) 2020. This article is licensed under a Creative Commons Attribution 4.0 International License, which permits use, sharing, adaptation, distribution and reproduction in any medium or format, as long as you give appropriate credit to the original author(s) and the source, provide a link to the Creative Commons licence, and indicate if changes were made. The images or other third party material in this article are included in the article's Creative Commons licence, unless indicated otherwise in a credit line to the material. If material is not included in the article's Creative Commons licence and your intended use is not permitted by statutory regulation or exceeds the permitted use, you will need to obtain permission directly from the copyright holder. To view a copy of this licence, visit http://creativecommons.org/licenses/by/4.0/. The Creative Commons Public Domain Dedication waiver (http://creativecommons.org/publicdomain/zero/1.0/) applies to the data made available in this article, unless otherwise stated in a credit line to the data. 
Conclusion: The bioinformatics data and in vivo experimental results suggest that the MXD-induced amelioration of NAFLD may be predominantly related to modulation of lipid metabolism and inflammation. Both BC and SM serve as the key role in MXD against NAFLD. These results may provide novel evidence for clinical implication of MXD.

Keywords: Modified Xiaochaihu Decoction, Non-alcoholic fatty liver disease, Network pharmacology

\section{Background}

Increased human longevity has led to a global burden of chronic diseases, such as cardiovascular disease, neurodegenerative diseases, metabolic syndromes, and most cancers $[1,2]$. Current data strongly indicates that ageing leads to non-alcoholic fatty liver disease (NAFLD), which is a consequence of metabolic syndrome [3]. The sum effect of age-related changes can contribute to an incremental increase in the susceptibility to development of NAFLD [4]. However, there is no available clinical drug approved [5], which underscores the pressing need for pharmacotherapy.

Xiaochaihu Decoction (XCHD) is a famous prescription first recorded in Shang Han Za Bing Lun [6]. From its origin in China to its spread into Japan and southeast Asia [7], there is a long history for XCHD as a traditional Chinese medicine to treat influenza, fever, and hepatitis [8]. It is composed of Pinellia ternate (Thunb.) Breit. (PT), Panax ginseng C. A. Mey. (PG), Glycyrrhiza uralensis Fisch. (GU), Scutellaria baicalensis Georgi. (SB), Zingiber officinale Rose. (ZOR), Ziziphus jujube Mill. (ZJ), and Bupleurum chinense DC. (BC) (Table 1). XCHD has been reported to achieve substantial curative effects in treating NAFLD in both clinical trials and animal studies. A clinical trial has demonstrated that XCHD supplementation results in a significant reduction in hepatic steatosis grade, total cholesterol (TC), triglyceride (TG), low-density lipoprotein cholesterol (LDL), alanine transaminase (ALT), and aspartate transaminase (AST) in patients with NAFLD [9]. Another clinical study on 84 cases reported an increased plasma adiponectin concentration and decreased body mass index (BMI), TC, TG, and controlled attenuation parameters (CAP) in NAFLD patients who received XCHD combined with lifestyle intervention $[10,11]$. XCHD can ameliorate high-fat dietinduced fatty liver and suppress hepatic de novo lipogenesis in $\mathrm{db} / \mathrm{db}$ mice $[7,12]$. However, these studies still focused on "single target and single pathway." A holistic "multiple compounds, multiple targets, and multiple pathways" study is necessary to clarify how XCHD produces liver-protective effect on NAFLD.

Several studies have demonstrated the protective effect of XCHD against NAFLD [9-12]. However, in traditional Chinese medicine (TCM) theory, the patients with NAFLD show many different kinds of syndromes. Therefore, Modified Xiaochaihu Decoction (MXD) has been extensively used in treating NAFLD in animal experiments and clinical trials [13-16]. Given that most of the patients with NAFLD experience the syndrome of spleen afflicted with sputum dampness, liver-qi stagnation, and turbid stasis obstructing collaterals, our research team made MXD by adding Salvia miltiorrhiza Bge. (SM), Artemisia scoparia Waldst. Et Kit (AS), and Curcuma wenyujin $\mathrm{Y}$. $\mathrm{H}$. Chen et $\mathrm{C}$. Ling $(\mathrm{CW})$ on the basis of XCHD (Table 1). SM is a kind of blood-activating and stasis-resolving medicine. It can potentially be beneficial to enhance the descending turbid effects of XCHD. SM has been used for centuries to treat liver diseases [17]. Several in vivo studies have indicated that SM alleviates hepatic inflammation, fatty degeneration, and hepatic fibrogenesis in NAFLD models [18]. The medicinal properties of AS are bitter, pungent and slightly cold. The function of $\mathrm{CW}$ in the perspective of TCM is to activate blood, relieve pain, promote qi, disperse the stagnated qi, clear heart, cool blood, excrete bile, and disperse jaundice. AS can boost the effects of XCHD on soothing the liver and strengthening the spleen in Herbal Prescription Science. AS extract attenuates NAFLD in mice with diet-induced obesity by intensifying hepatic insulin and MAPK signalling pathway [19]. CW can promote liver-qi, disperse stagnated qi, excrete bile, and disperse jaundice. It is added in XCHD to magnify the efficacy of soothing the liver, descending turbid, and resolving phlegm. CW has a hepatoprotective and combinatory preventive effect with silymarin on methionine choline deficient-dietinduced NAFLD in C57BL/6J mice [20]. As a matter of fact, XCHD still plays a major role in MXD, while the other three herbs may enhance the treatment effect of $\mathrm{XCHD}$ on soothing the liver, strengthening the spleen, descending turbid, and resolving phlegm. Our clinical trials for many years have shown that it is appropriate for most patients with NAFLD. Here, we investigate the therapeutic effects of MXD by network pharmacology and experimental validation.

Network pharmacology has been proved to be an effective method to explore potential targets and pathways of TCM by analyzing network of biological systems. The systematism of the strategy resonates well with the holistic view of TCM, as well as the mechanism of multiingredient, multi-pathway, and multi-target synergy in TCM formulas [21]. Many studies have demonstrated that TCM network pharmacology approach provides 


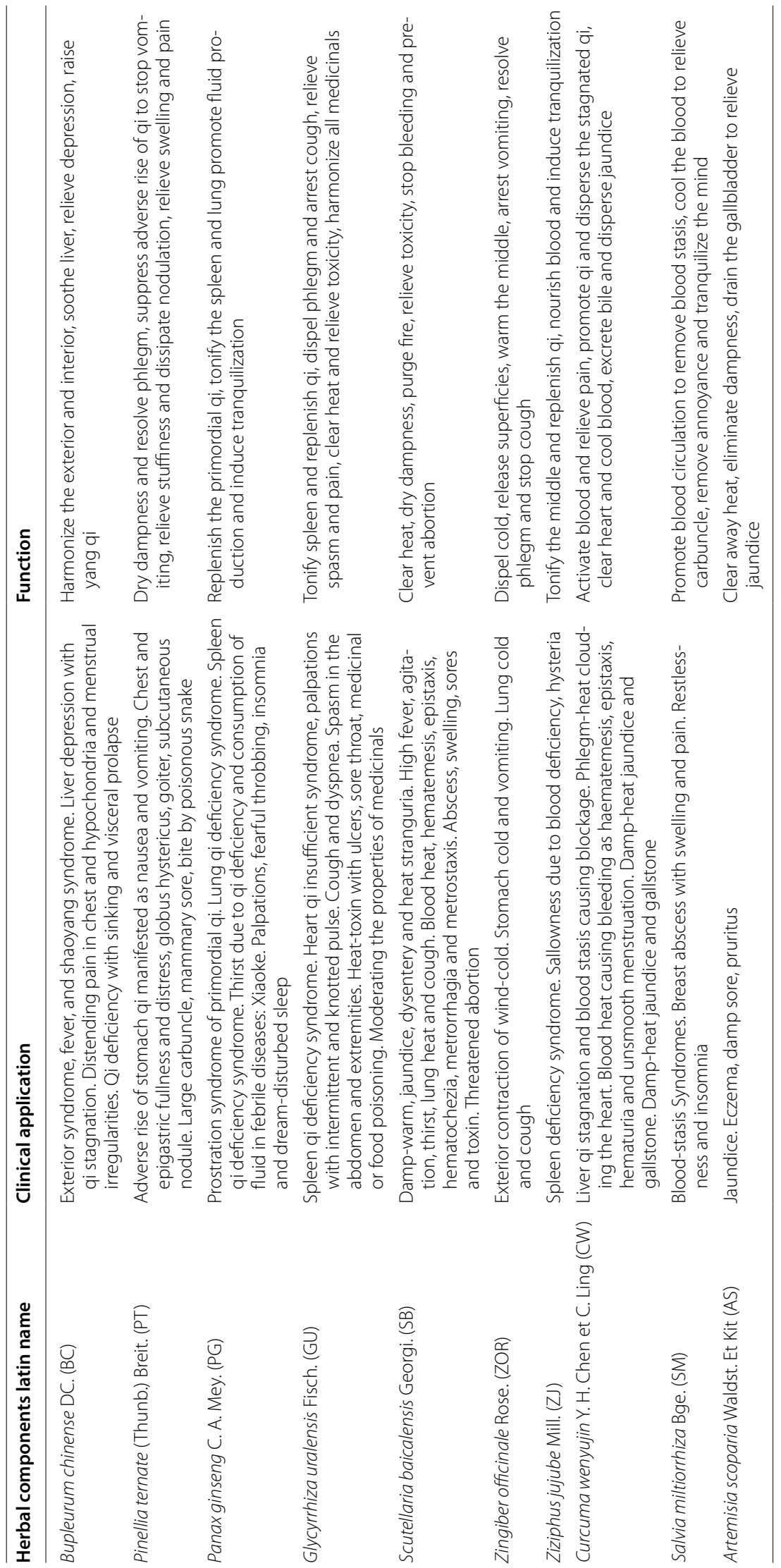


a new research paradigm for translating TCM from an experience-based medicine system to an evidence-based medicine system, which will accelerate TCM drug discovery and improve current drug discovery strategies [22-24]. In this study, we first predicted the potential targets of MXD involved in NAFLD. Then, ageing rats were used as an appropriate animal model to verify the improvement effects of MXD on ageing-related NAFLD, to validate some of the potential targets, and to distinguish the auxiliary and major herbs of MXD (Fig. 1).

\section{Methods}

\section{Data preparation}

A catalogue of chemical ingredients of ten herbs in MXD was generated from related literature and the Traditional Chinese Medicines systems pharmacology database (TCMSP, https://lsp.nwu.edu.cn/tcmsp.php). TCMSP is a system pharmacology platform for users to comprehensively study TCM via the screening of drug targets, including identification of active components and generation of compounds-targets-diseases networks. The targets of candidate molecules were also collected from the TCMSP database. To be specific, they were collected from the Related Targets of ingredients section in TCMSP (https://tcmspw.com/tcmsp.php).

Data on the NAFLD-related targets were obtained from three database: (1) Gene Cards (Gene Cards ${ }^{\circledR}$ : The Human Gene Database, https://www.genecards.org/) is a human gene database that provides comprehensive, user-friendly information on annotated and predicted human genes. (2) Online Mendelian Inheritance in Man (OMIM, https://omim.org/) is an authoritative, comprehensive compendium of human genes and genetic phenotypes. (3) Genetic Association Database (GAD, https:// geneticassociationdb.nih.gov/) is a human gene database which provides genes associated with complex diseases and disorders. We searched these databases with the keywords "non-alcoholic fatty liver disease" and "NAFLD".

\section{Data pretreatment}

The active ingredients of $\mathrm{BC}, \mathrm{PT}, \mathrm{PG}, \mathrm{GU}, \mathrm{SB}, \mathrm{ZJ}, \mathrm{ZOR}$, $\mathrm{SM}, \mathrm{AS}$, and $\mathrm{CW}$ were screened out with the standards of $\mathrm{OB} \geq 20 \%$ and $\mathrm{DL} \geq 0.1$ provided by numerous literatures [25]. Potential targets corresponding to all ingredients were collected first, then the targets corresponding to active ingredients of each herb were screened out.

\section{Network construction}

Interaction of potential NAFLD targets with the targets corresponding to active ingredients of MXD was presented by VENN diagram. The graphical interactions were visualized using Cytoscape software (https://cytos cape.org/, ver.3.6.0).
Protein-protein interaction (PPI) network analysis, pathway and functional enrichment analysis

PPI network analysis is a network structure that presents the relationship of proteins work with other molecules, such as other proteins, lipids, and nucleic acids. The data source came from STRING 11.0 (https://string-db.org/ cgi/input.pl?taskId=_notask\&sessionId=Uj9LD8q5bY $\mathrm{kw}$ ). Data was analyzed by EXCEL software (Microsoft Office Professional Plus 2016). Each node in the PPI network analysis was assessed with its eight typical attributes in STRING: text mining, co-expression, protein homology, gene neighborhood, gene fusions, gene cooccurrence, from curated databases, and experimentally determined. The minimum required interaction scores of edges in PPI network is 0.4 (which is medium confidence). The research species was defined as "Homo sapiens". The rest of the parameters were set to the default in STRING. Data of Gene Ontology (GO) functional enrichment analysis was obtained from the Database for Annotation Visualization and Integrated Discovery (DAVID 6.8, https://david.nicifcrf.gov/). Kyoto Encyclopedia of Genes and Genomes (KEGG) Pathway enrichment analysis was performed based on the $\mathrm{R}$ clusterProfiler package [26].

\section{Animals, diet, and experimental design}

Female young (3-month) and old (20-month) Wistar rats were purchased from Laboratory Animal Centre of the Army Medical University, China. Then, rats were maintained in the Laboratory Animal Centre of Chongqing Medical University, China. All experimental procedures were carried out according to internationally accepted principles for laboratory animal use and care and were approved by the Animal Ethics Committee, Chongqing Medical University, China. The rats were kept in a thermostatic-control facility at $21 \pm 1{ }^{\circ} \mathrm{C}, 55 \pm 5 \%$ relative humidity under a $12 \mathrm{~h}$ light/dark cycle. All rats were acclimated for 1 week prior to beginning the experiment.

Sixteen ageing rats were divided initially into two groups ( $\mathrm{n}=8$ per group): the natural ageing group (ageing) and the group (MXD) supplemented with MXD at $10.8 \mathrm{~g} / \mathrm{kg}$. Eight young female rats were randomly selected as the young normal group $(\mathrm{NC}, \mathrm{n}=8)$. All rats had free access to water and standard chow diet. Rats in the MXD-treated groups received $10.8 \mathrm{~g} / \mathrm{kg}$ MXD (suspended in 5\% Gum Arabic solution and provided by oral gavage once daily) for 5 weeks. The rats in the ageing and NC groups received vehicle ( $5 \%$ Gum Arabic) alone. At the end of week 4, blood samples were collected via retroorbital venous puncture under isoflurane anesthesia after an overnight fast (14 h) to measure plasma TG, TC, glucose, and insulin concentration. At the end of week 5 , the rats were deprived of chow, but still had free access 


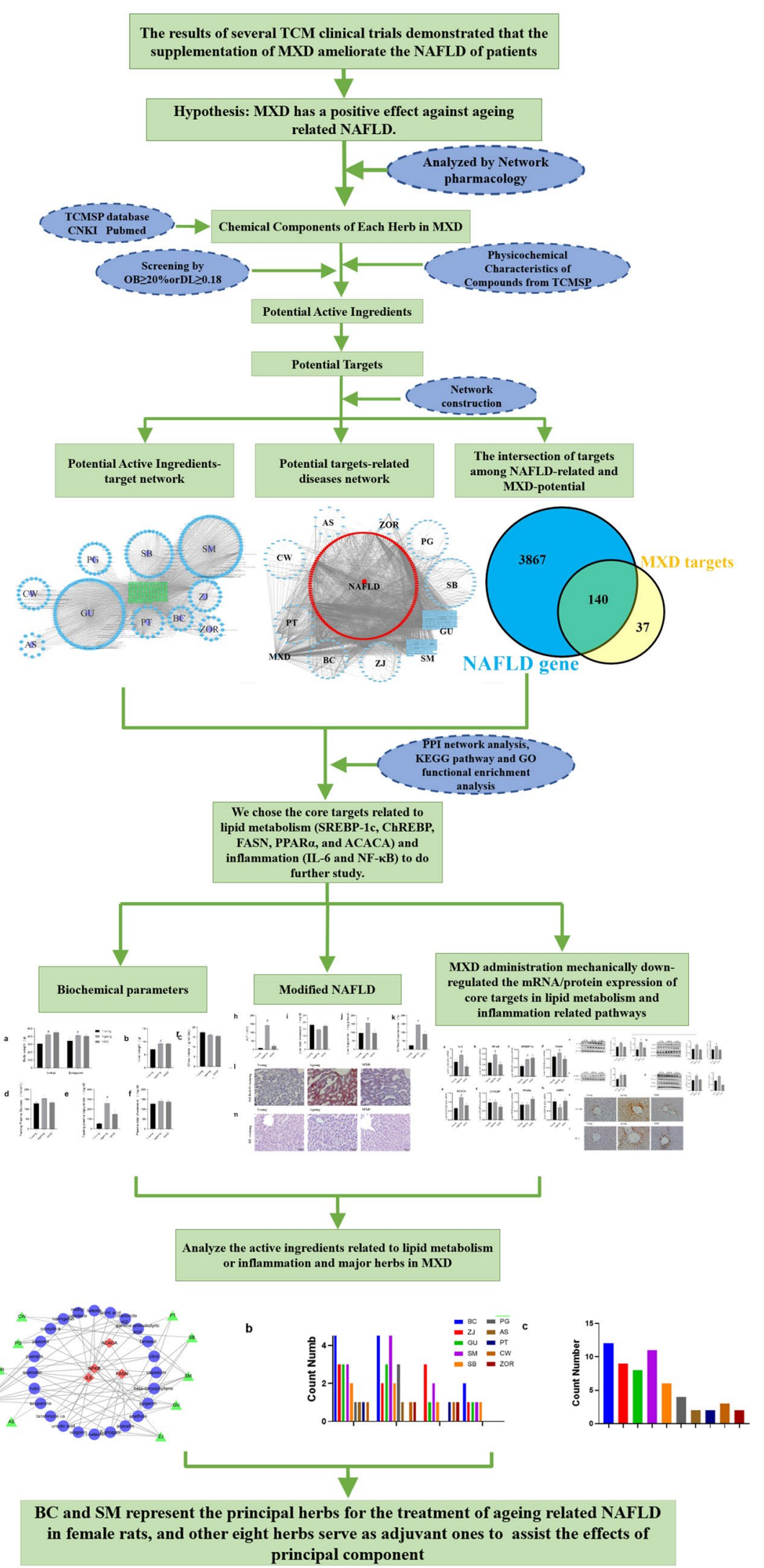

Fig. 1 Study design 
to water overnight $(14 \mathrm{~h})$. Then, the rats were weighed, deeply anaesthetized with isoflurane, and sacrificed by prompt cervical dislocation. The livers were removed, weighed, immediately frozen in liquid nitrogen, and stored at $-80{ }^{\circ} \mathrm{C}$ until use.

\section{Blood and liver biochemical analysis}

Fasting plasma TG, TC, glucose, ALT, and AST were estimated using commercially available kits and based on the manufacturer's instructions (kits A110-1-1, A111-11, F006-1-1, C009-2-1, and C010-2-1, respectively; Nanjing Jiancheng Bioengineering institute, Nanjing, China). One hundred mg of liver homogenate was extracted with $2 \mathrm{~mL}$ isopropanol. After centrifugation at $3000 \mathrm{rpm}$, the contents of triglyceride and total cholesterol in supernatant were estimated by enzymatic method (A110-1-1 and A111-1-1 kits, respectively; Nanjing Jiancheng Bioengineering Institute, Nanjing, China).

\section{Histological examination}

A portion of liver was fixed with $10 \%$ formalin and embedded in paraffin. Four-micron sections were cut and stained with hematoxylin and eosin (HE) for examination of liver histology.

Appropriate size liver was fixed with $10 \%$ paraformaldehyde and embedded in OCT embedding medium. Oil red $\mathrm{O}$ staining was performed on six-micron frozen sections with a freezing microtome. The Oil Red O-stained area as well as the total tissue area in each field were measured using Image J software (Version 1.52t 30 January 2020 upgrade). The ratio of the Oil Red O-stained area to the total tissue area was calculated (\%).

\section{Real-time PCR}

Total RNA was extracted from the livers using TRIzol (Takara, Dalian, China) following the manufacturers procedure. cDNA was synthesized by employing an M-MLV R-Tase cDNA Synthesis Kit (Cat\# RR037A; Takara, Dalian, China). Real-time PCR was performed with a CFX 96 Real-time PCR Detection System (Bio Rad Laboratories Inc., Hercules, CA, USA) using the SYBR ${ }^{\circledR}$ Premix Ex Taq ${ }^{\text {TM }}$ II (Takara, Dalian, China).

\section{Western blots analysis}

Frozen liver tissues were homogenized in radio-immunoprecipitation assay (RIPA) buffer with 1\% PMSF to produce a total extract. Proteins were separated via SDSPAGE and were transferred to a polyvinylidene difluoride (PVDF) membrane (Millipore Corporation, Billerica, USA) by electro-blotting. The membranes were blocked with $5 \%$ skim milk for $90 \mathrm{~min}$ at room temperature and then incubated with the primary antibodies overnight at $4{ }^{\circ} \mathrm{C}$. After incubation with appropriate secondary antibodies, detection of signals was performed using ECL Western blot detection kit (Pierce Biotechnology, Rockford, IL, USA). The density was evaluated using the Image Lab software (version 5.2.1, Bio-Rad Laboratories, California, HE, USA). The amount of protein expression was normalized to the amount of $\beta$-actin in the same sample.

\section{Immunohistochemistry}

Four micron serial paraffin sections were processed for immunohistochemical staining with a monoclonal antibody against NF-kB (Cat\# ab216409; dilution 1:1000, Abcam, Cambridge, MA, USA) and IL-6 (Cat\# ab6672; dilution 1:1000, Abcam, Cambridge, MA, USA). Sections were incubated with goat anti-rabbit and goat anti-mouse secondary antibody, respectively. Next, the samples were submitted to two-step assay kit (Cat\# PV-9002; Zhongshan Golden Bridge Biotechnology, Beijing, China), followed by incubation with DAB kit (Cat\# ZLI-9018; Zhongshan Golden Bridge Biotechnology, Beijing, China) [27]. The images of the sections from each group were acquired with a light microscope at a magnification of $400 \times$.

\section{Data analysis}

All results were expressed as means $\pm S E M$. Data were analyzed by ANOVA using the GraphPad Prism 8 software, followed by The Student-Newman-Keuls test to determine the differences between groups. $\mathrm{P}<0.05$ was considered statistically significant.

\section{Results}

Potential ingredients and targets in MXD

A total of 1953 chemical ingredients of MXD were obtained from the TCMSP database (Fig. 2a) and related literature (Fig. 2b) in 10 herbs: BC, PT, PG, GU, SB, ZOR, $\mathrm{ZJ}, \mathrm{SM}, \mathrm{AS}$, and $\mathrm{CW}$. The ingredients were screened according to the criteria of $\mathrm{OB} \geq 20 \%$ and $\mathrm{DL} \geq 0.1$. Among the 1953 chemical ingredients in MXD, 1350 $(69.12 \%)$ met the requirement of $\mathrm{OB} \geq 20 \%$ and 617 (31.59\%) met the requirements of $\mathrm{OB} \geq 20 \%$ and $\mathrm{DL}$ index $\geq 0.1$. Of these 617 bioactive ingredients, 30 (4.86\%) from PT, 66 (10.70\%) from PG, 125 (20.26\%) from GU, 56 (9.08\%) from SB, 39 (6.32\%) from ZOR, 52 (8.43\%) from ZJ, 56 (9.08\%) from BC, 97 (15.72\%) from SM, 17 (2.76\%) from AS, and 79 (12.80\%) from CW (Fig. 2c). The details of these ingredients are shown in Additional file 1: Table S1. A total of 177 potential pathophysiological targets of these chemical ingredients were collected from the TCMSP database. To further understand the MXD component-targets network from a holistic and systematic point-of-view, we built a network map (Fig. 2d). It contains 804 nodes (617 for bioactive ingredients, 10 for 


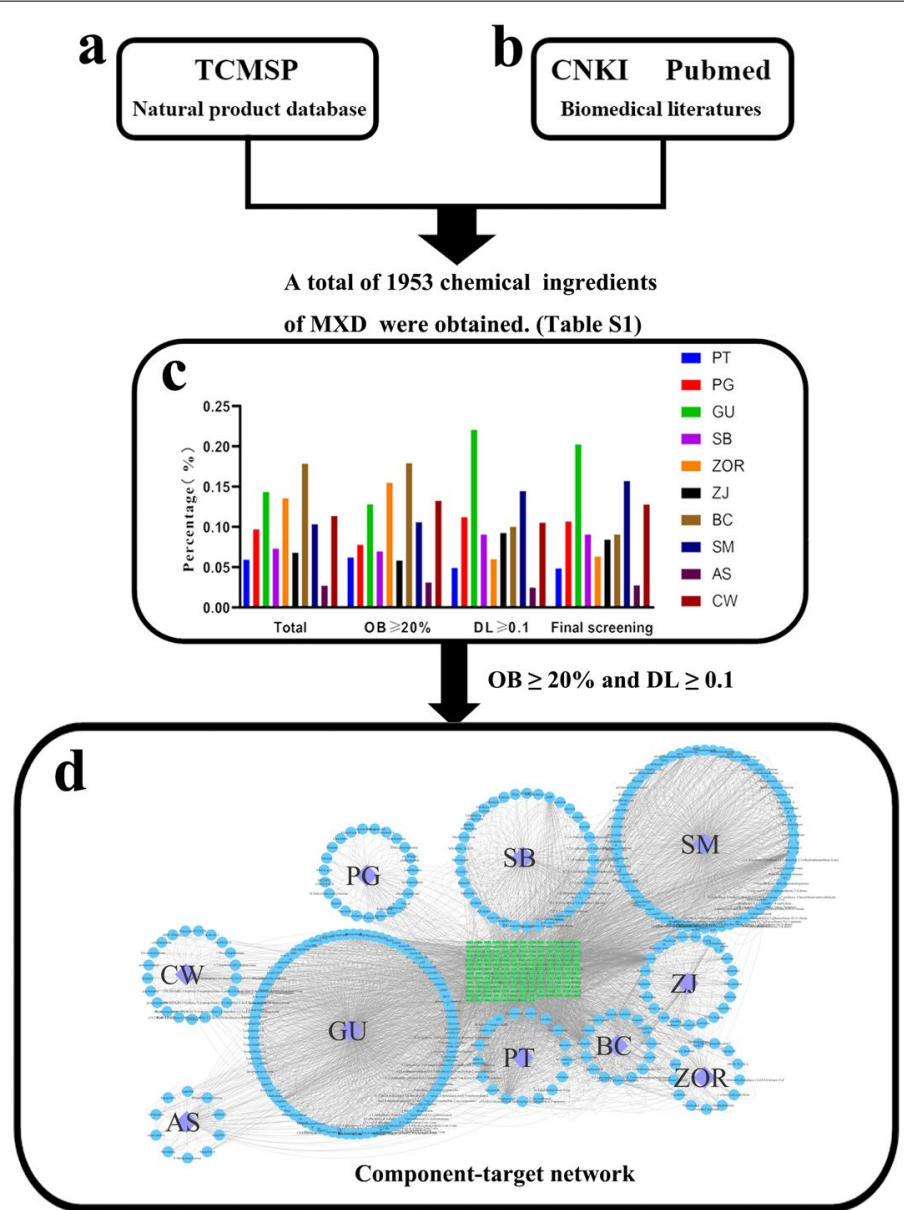

Fig. 2 MXD component-targets network. Two broad categories database: a natural product databases. b Biomedical literatures. $\mathbf{c}$ Distributions of different herbs $\mathbf{d}$ Construction of MXD bioactive component-putative targets visual network, containing 804 nodes and 15,468 edges. The blue dots represent putative bioactive components of MXD. The purple diamond nodes represent herbs. The green elliptic nodes represent potential targets related to putative bioactive components of MXD

herbs in MXD, and 177 for potential targets). The edges represent the interaction between the active ingredients and potential targets or the herbs in MXD and active ingredients. Detailed information on the active ingredients and potential pathophysiological targets is presented in Additional file 2: Table S2.

\section{NAFLD-related target network}

We obtained 259 NAFLD-related targets from OMIM, 989 NAFLD-related targets from GAD and 3763 NAFLDrelated targets from GeneCards (Fig. 3a). The details of these NAFLD-related targets are shown in Additional file 3: Table S3. After removing duplicates, total 4009 NAFLD-related targets were collected (Fig. 3b). Among this, MXD shared 140 common targets with NAFLD (Fig. 3c). To gain insights into the pharmacological mechanisms of MXD on NAFLD, we constructed a common target network (Fig. 3d).

\section{PPI network analyses}

To further investigate the core pharmacological mechanisms of the effects of MXD in treating NAFLD, we applied a topological method to evaluate the core network. PPI network analysis is beneficial to understanding the role of various protein in the complex pathological mechanisms of NAFLD. Therefore, we established an interactive PPI network of MXD and NAFLD (Fig. 4a). In the network, 138 targets can have protein interaction (Two targets do not have protein interaction). 1775 edges represent the interaction between proteins. The number of edges between targets means the degree of nodes. After statistical analysis of the PPI network of MXD and NAFLD, we choose 30 core targets based on degree (Fig. 4b), including serum albumin (ALB), Interleukin-6 (IL-6), vascular endothelial growth factor A (VEGFA), epidermal growth factor receptor (Egfr), Caspase-9 (Casp3), Sterol regulatory element-binding protein 1 
Fig. 3 NAFLD-related targets network. a Three disease gene target databases. b NAFLD-target network, containing 4008 nodes and 4007 edges. The purple nodes represent targets related to NAFLD. The yellow node represents NAFLD. c 140 common targets between NAFLD and MXD. d Common target network, containing 453 nodes and 2877 edges. Red dots represent common targets of MXD and NAFLD. Blue square nodes represent putative bioactive components of MXD. Purple node represents the prescription (MXD) we use. Orange node represents the disease (NAFLD). Edges stand for the interactions among putative bioactive components of MXD, NAFLD, targets and MXD

(SREBP-1c), peroxisome proliferators-activated receptor alpha $(\mathrm{PPAR} \alpha)$, Mitogen-activated protein kinase 8 (MAPK8), RELA Proto-Oncogene ( RelA, also known as Nuclear factor kappa- $\beta$ Subunit, NF-k $\beta$ P 65 , and NF- $\kappa \beta$ ), Fatty acid synthase (FASN), and other targets. It is speculated that these targets may be the key targets for MXD in the treatment of MXD.

\section{GO functional enrichment analyses}

GO functional (including molecular functions, biological process, and cellular component) enrichment analysis are the common methods used to describe the characteristics of candidate targets. The detailed GO function information are shown in Additional file 4: Table S4. There were respectively 112 biological process, 23 cellular component, and 32 molecular function terms in total, which met the requirements of count $\geq 2$ and P-value $\leq 0.05$. The top 20 significantly enriched terms in molecular functions, biological process, and cellular component categories were shown in Fig. 4c. The top 20 terms with the largest number of targets in molecular functions, biological process, and cellular component were shown in Fig. 4d.

Among them, we found triglyceride metabolic process (GO:0006641), fatty acid biosynthetic process (GO:0006633), cholesterol homeostasis (GO:0042632), cholesterol metabolic process (GO:0008203), steroid binding (GO:0005496), steroid hormone receptor activity (GO:0003707), lipid transporter activity (GO:0005319), chromatin binding (GO:0003682), fatty acid binding (GO:0005504), and triglyceride homeostasis (GO:0070328) are closely related to lipid metabolism. Likewise, inflammatory response (GO:0006954), positive regulation of interleukin-12 biosynthetic process (GO:0045084), positive regulation of I-kappaB kinase/NF-kappaB signaling (GO:0043123), and positive regulation of NF-kappaB transcription factor activity (GO:0051092) are related to inflammation. Notably, consistent with this result, numerous targets appear in the PPI analysis also involved in lipid metabolism (such as SREBP-1c, FASN, PPAR $\alpha$, and ACACA) and inflammation (such as IL-6 and NF-kB).

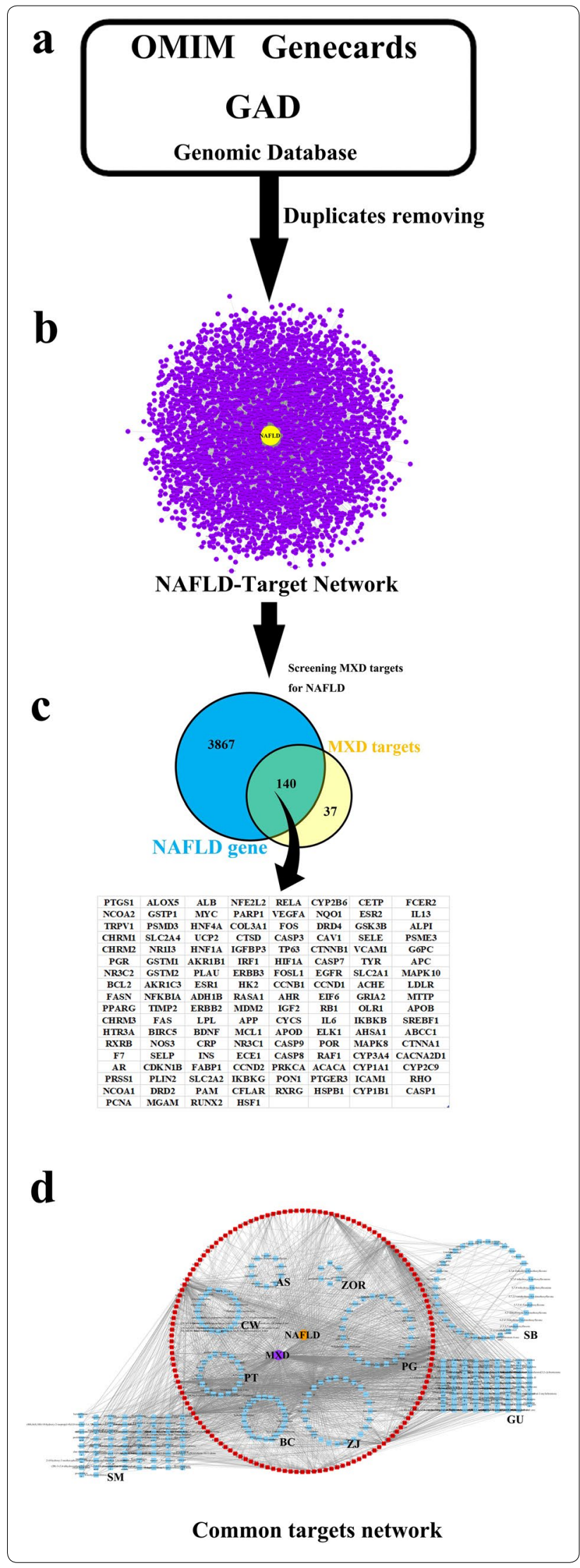


(See figure on next page.)

Fig. 4 Construction of MXD-NAFLD PPI network. a Interactive PPI network of MXD and NAFLD, containing 138 nodes and 1775 edges. The node size is proportional to the target degree in the network. The node color changes from orange to blue reflect the degree value changes from low to high in the network. b Thirty core targets in PPI network arranged in order of degree. c GO functional analysis: the top 20 significantly enriched $\mathrm{GO}$ terms in molecular functions, biological process, and cellular component. $\mathbf{d}$ The top 20 terms with the largest number of targets in molecular functions, biological process, and cellular component

\section{KEGG pathway enrichment analysis}

To clarify the underlying mechanisms of the action of MXD for treatment of NAFLD, a pathway enrichment analysis was performed based on KEGG database. We fed the clusterprofile $\mathrm{R}$ package [26] a list of major gene targets to generate relevant pathways that might have an important influence on the amelioration by MXD of NAFLD. Only pathways with P-value $<0.05$ were considered as significant ones. The detailed information on the KEGG pathway enrichment analysis is presented in Additional file 5: Table S5. The results demonstrated that 140 targets were mapped into 131 KEGG pathways, included in prostate cancer, fluid shear stress and atherosclerosis, and hepatitis $\mathrm{B}$. We analyzed the data and relevant biological processes, selected top 20 significant pathways according to the GeneRatio (Fig. 5a) and P-value (Fig. 5b).

To further characterize the molecular mechanism by which MXD alleviated NAFLD, a target-pathway network was performed based on all involved proteins and their corresponding significant signalling pathways (Fig. 5c). This network included 253 nodes (122 for proteins and 131 for pathways). The edges represent the interactions between targets and pathways. The number of edges between targets means the degree of nodes. Among these potential targets, RelA (NF-KB), IL-6, MAPK10, SREBF1, PPAR $\alpha$, RAF1, MYC, MAPK8, IKBKG, CCND-1, and CASP3 were identified as relatively high-degree targets.

Consolidated researches indicate that lipid metabolism, oxidative stress, and inflammation may play a critical role in the development of NAFLD [28]. Evidence has been found that the progression of NAFLD has been associated with marked alterations in hepatocyte histology and a shift in marker expression of healthy hepatocytes including inflammation and lipid metabolism [29].

Taken together, based on the results of PPI analysis, GO functional enrichment analysis, KEGG pathway enrichment analysis, and current research on the mechanism of NAFLD, we plan to examine the effect of MXD on the improvement of ageing-related NAFLD and to mechanistically verify the change of core targets related to lipid metabolism (SREBP-1c, PPAR $\alpha$, FASN, and ACACA) and inflammation (IL-6 and NF- $\mathrm{KB}$ ).

In addition, we found that many diseases such as Alzheimer disease, measles, atherosclerosis, hepatitis, cancer, and other related biological molecules such as prolactin, thyroid hormone, and insulin could also indirectly affect the development of NAFLD, providing strong evidence for our hypothesis that MXD could treat NAFLD through a "multiple compounds, multiple targets, and multiple pathways" way.

\section{MXD ameliorated plasma TG and hepatic steatosis in ageing female rats}

Ageing rats had significantly higher body weight (Fig. 6a) and liver weight than young rats (Fig. 6b), but tended to have a decreased chow intake (Fig. 6c). MXD treatment did not affect these parameters (Fig. 6a-c).

Compared with Young group, plasma concentrations of TG (Fig. 6d), AST (Fig. 6e), and ALT (Fig. 6f) were elevated in Ageing group. Significantly, MXD treatment reversed these changes. However, there was no change in the plasma TC (Fig. 6g) and glucose concentration (Fig. 6h) among the groups.

Hepatic TG content was obviously increased (Fig. 6i) in ageing rats. However, there was no change in the hepatic TC content among groups (Fig. 6j). Consistent with this finding, the increase in vacuolization (Fig. 6l) and the ratio of Oil Red O-stained area (Fig. 6k, m) to total tissue area was evident on histological examination of liver sections from ageing rats compared with young rats, which was indicative of excess lipid droplet accumulation. MXD treatment significantly decreased hepatic TG content (Fig. 6i), vacuolization (Fig. 61), and Oil Red O staining area (Fig. 6 k, m).

\section{Molecular mechanism of MXD in alleviating hepatic steatosis in ageing female rat}

Using the preliminary data analyses as a guide, we further explored the molecular mechanism of MXD alleviating hepatic steatosis in ageing female rats. First, we examined IL- 6 and NF- $\kappa \beta$. Both of them are important targets in the preliminary network pharmacology analysis. As predicted, ingestion of MXD significantly decreased mRNA/protein expression of IL-6 (Figs. $7 \mathrm{a}, 8 \mathrm{a}, \mathrm{f}$ ) and NF-kB (Figs. 7b, 8a, e). Then we examined Interleukin-1 $\beta$ (IL-1 $\beta$ ) and Tumor necrosis factor alpha (TNF $\alpha)$. They are key mediators of the inflammatory response and closely related to NF- $\mathrm{kB}$, ingestion of MXD significantly decreased protein expression of IL-1 $\beta$ (Fig. 8d) and TNF- $\alpha$ (Fig. 8d). 


\section{a}

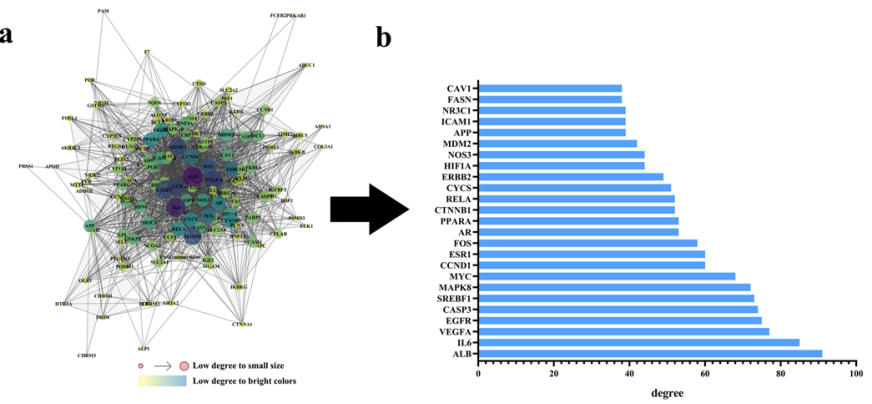

b

c

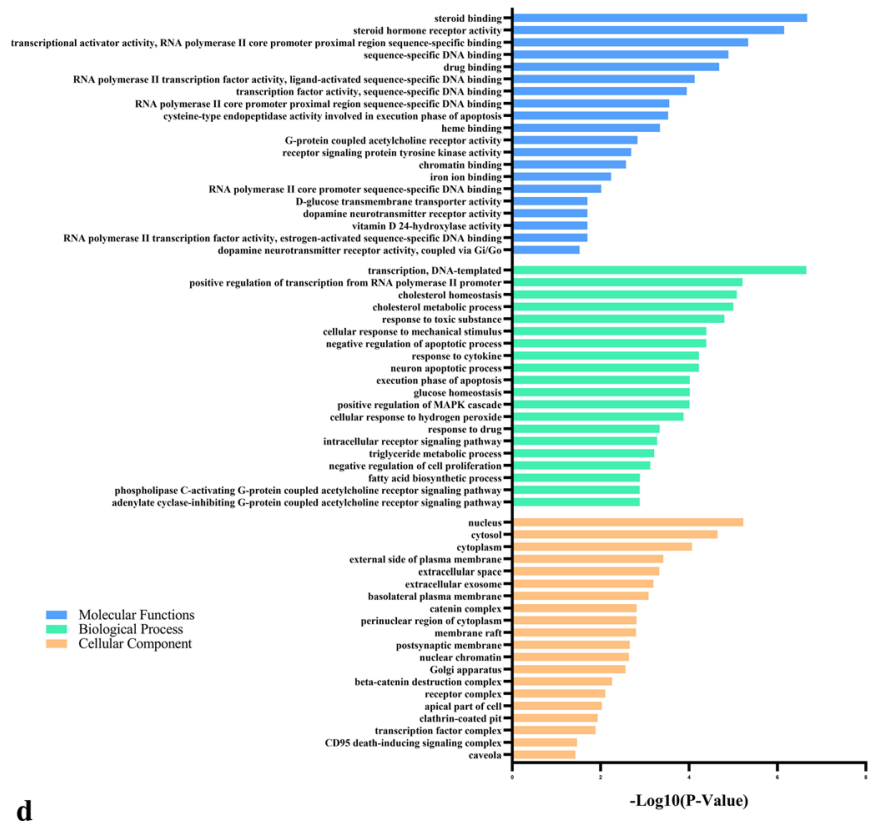

d

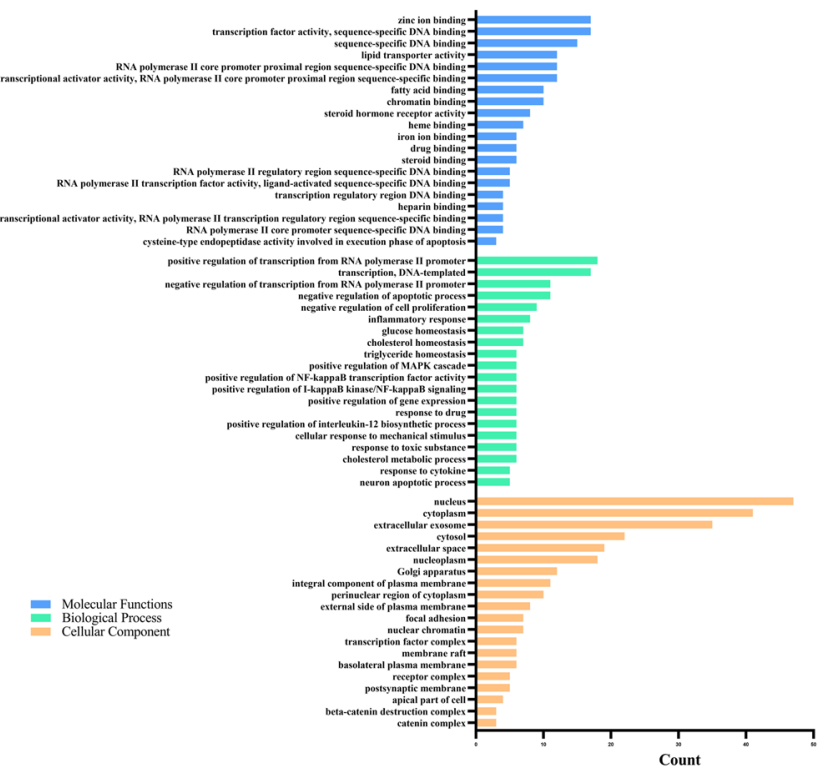



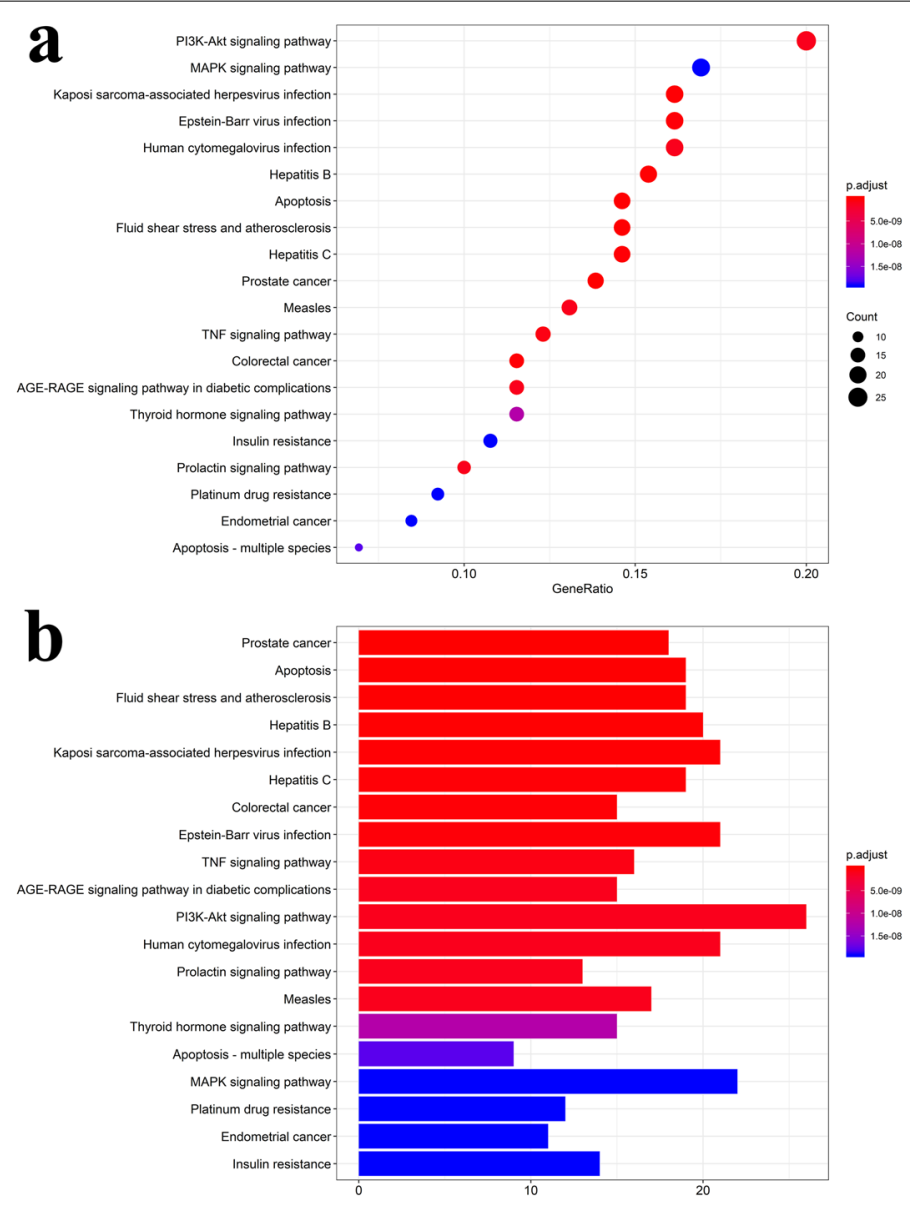

C

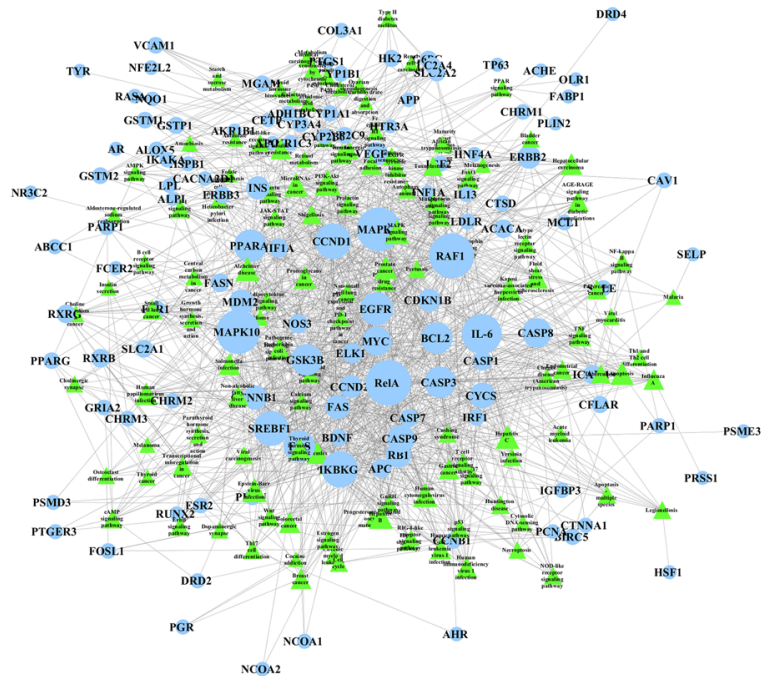

Fig. 5 Top 20 putative signalling pathway in KEGG pathway enrichment analysis by a GeneRatio and $\mathbf{b}$ P-value. c The target-pathway network for MXD on NAFLD, containing 253 nodes and 1328 edges. The blue nodes represent targets and the green nodes represent pathways. The edges represent the interactions between them and node size is proportional to their degree 
Furthermore, regarding the pathway involved in hepatic de novo lipogenesis, MXD decreased the level of gene and protein expressions of Acetyl-CoA carboxylase 1 (ACACA) (Figs. 7e and 8b) and Fatty acid synthase (FASN) (Figs. 7d and 8b), while mRNA/protein expression of Sterol regulatory element-binding protein 1c (SREBP-1c) (Figs. 7c and 8c).and carbohydrate response element binding protein (ChREBP) was changeless (Fig. 7f).

Moreover, MXD upregulated the expression of PPAR $\alpha$ mRNA (Fig. 7G) and AMPK mRNA (Fig. 7H), which are the master regulator of fat oxidation and energy homeostasis, respectively.

\section{Distinguish the auxiliary and major herbs of MXD}

Every herb in the formula has specific ingredients (Fig. 9a). Twelve of 24 potential ingredients acting on verified targets (IL-6, NF-kB, FASN, and ACACA) come from $\mathrm{BC}$ while eleven of 24 potential ingredients acting on verified targets come from SM (Fig. 9b), implying that both BC and SM serve as the key role in treating NAFLD.

There are 4 bioactive components (ursolic acid, quercetin, apigenin, and tanshinone iia) activating targets related to both lipid metabolism and inflammation pathways (Additional file 6: Table S6). Four herbs (BC, ZJ, GU, and $\mathrm{SM}$ ) in the formula have share the common ingredients acting on both lipid metabolism and inflammation related pathways (Fig. 9c). This combination probably has a broader effect at a lower concentration, and is evidently safer than a single drug.

\section{Discussion}

NAFLD is increasing rapidly owing to increased human longevity [1]. It is strongly associated with dyslipidemia, T2DM, obesity, and hypertension [30]. However, there are no effective drugs for treating NAFLD available in the clinic $[5,30]$. TCM plays an essential role in complementary and alternative medicine, and has markedly contributed to the therapeutic action of metabolic diseases $[31,32]$. Several hundred years of clinical practice have confirmed the efficacy of TCM. XCHD is a classic prescription with a long history of clinical applications [33]. MXD, containing seven commonly used herbs (BC, PT, PG, GU, SB, ZOR, and ZJ), is formulated using the TCM formulation of XCHD with the addition of another three herbal plants (SM, AS, and CW). Based on therapy with syndrome differentiation and the results of several Traditional Chinese medicine (TCM) Clinical studies [9-11], we preliminarily hypothesized the protective effect of MXD against NAFLD.

After the verification by pharmacology network analysis, we further recognized that MXD played a positive role in NAFLD and then predicted its active ingredients and potential mechanism. This provided clues to translate the ancient constructs of therapy into those used in modern medicine. Our study utilized naturally ageing female rats as a model demonstrating that MXD treatment can ameliorate ageing-related NAFLD.

The bioinformatics data elucidate that anti-NAFLD pharmacological activities of MXD may be predominantly related to the modulation of lipid metabolism and inflammation related signalling pathways. Specifically, IL- 6 and NF- $\mathrm{kB}$ are core targets in related significant pathways associated with inflammation. SREBP-1c, PPAR $\alpha, A C A C A, F A S N$ are core targets in related significant lipid metabolism pathways. Correspondingly, in vivo animal experimental results showed that MXD administration ameliorated age-related NAFLD and mechanically down-regulated the mRNA/protein expression of core targets in lipid metabolism and inflammation related pathways such as FASN, ACACA, IL-6, NF-kB, IL-1 $\beta$ and TNF $\alpha$.

It has been demonstrated that de novo hepatic lipogenesis is mediated by two important transcription proteins, ChREBP and SREBP-1c [34-36]. SREBPs transport from the endoplasmic reticulum to the Golgi apparatus, where the active nuclear isoform of SREBPs is cleaved by specific proteases, then translocate into the nucleus and become nSREBPs [37]. Therefore, the nSREBP-1c may regulate some of the key enzymes in fatty acid synthesis such as FASN and ACACA. Similarly, the localization of ChREBP in the nucleus is a key determinant for its functional activity [38]. In our present study, the mRNA and total protein expression of SREBP-1c and ChREBP mRNA in liver did not change significantly, but their downstream genes FASN and ACACA [37], did have a reduced expression induced by MXD, we suspect that MXD might affect SREBP-1c and/or ChREBP translocation from cytoplasm to nucleus to regulate downstream genes. Therefore, the expression of nSREBP-1c and $\mathrm{nChREBP}$ remain to be further clarified. Moreover, our research also found that MXD treatment increased mRNA level of AMPK and PPAR $\alpha$ in naturally ageing rat models, which indicates that MXD may ameliorate ageing-related NAFLD through regulating intracellular energy metabolism and fatty acid oxidation.

After confirming the improvement effect of MXD on NAFLD and preliminarily exploring the potential molecular mechanism based on network pharmacology, we further speculated the role of different herbs in contribution to the achievement of MXD.

The results indicate that both $\mathrm{BC}$ and SM share the most bioactive components related to lipid metabolism and inflammation related pathways of the ten herbs in MXD, while the other eight herbs share less. Further integrated network shows that $\mathrm{BC}$ and $\mathrm{SM}$ represent 


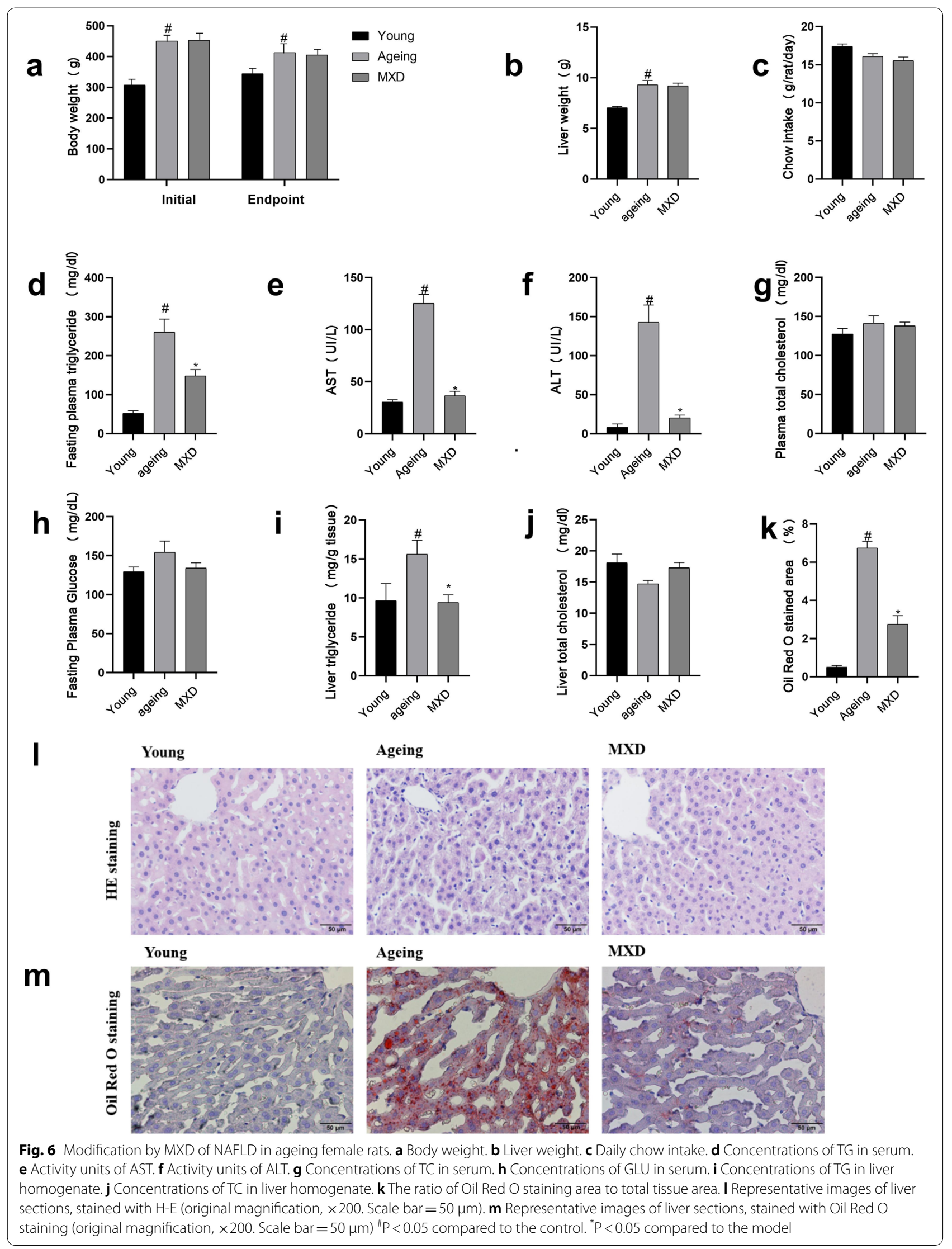



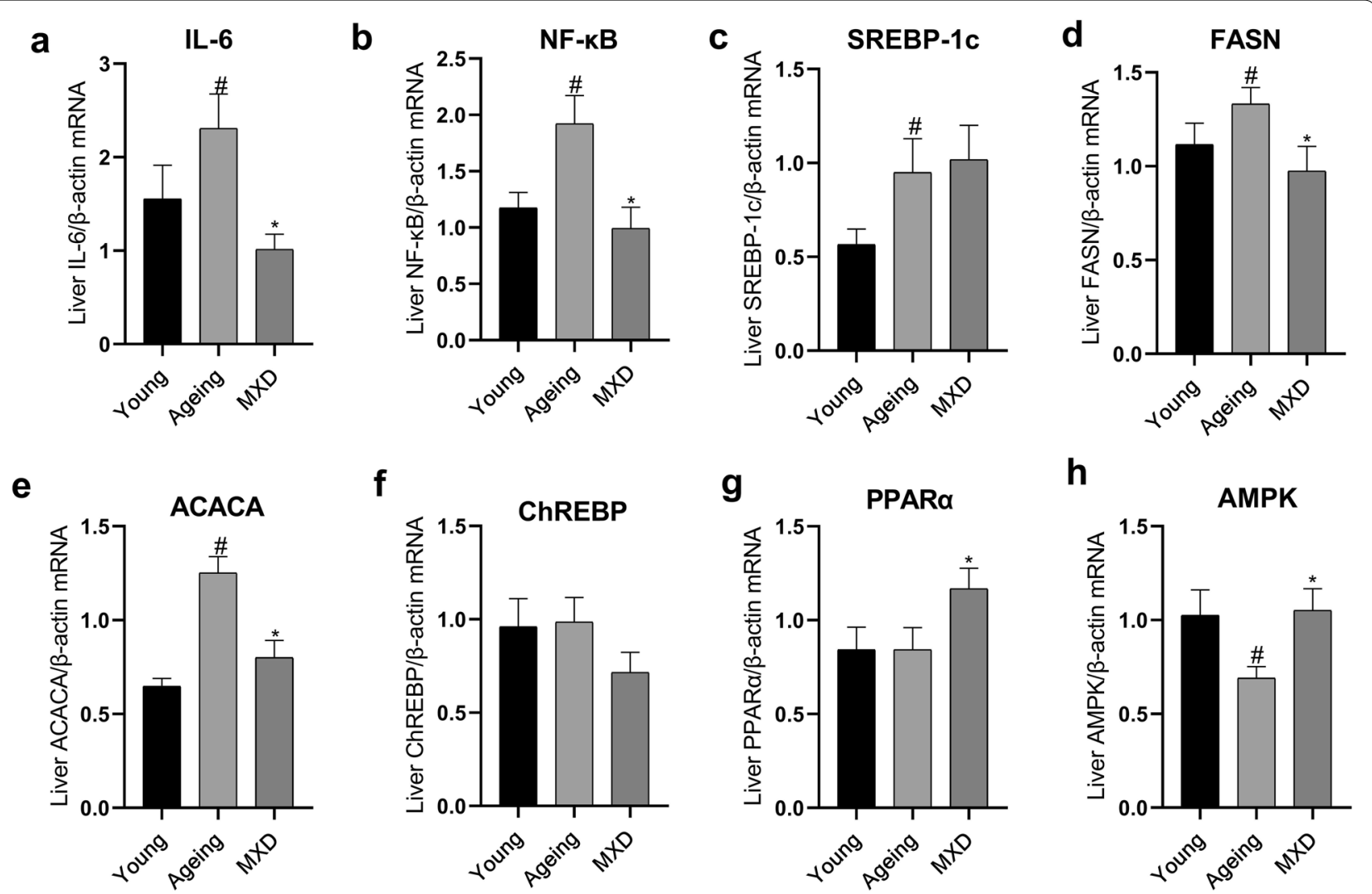

Fig. 7 Effect of MXD against NAFLD on the mRNA expression of inflammatory and metabolic genes in ageing female rats. a-h The mRNA expression of IL-6, NF-KB, SREBP-1C, FASN, ACACA, ChREBP, PPARa, and AMPK were determined by RT-PCR analyses. $\beta$-actin was as the internal reference. ${ }^{\#} \mathrm{P}<0.05$ compared to the control. ${ }^{*} \mathrm{P}<0.05$ comped to the model

the principal herb for the treatment of ageing-related NAFLD in female rats, and the other eight herbs serve as adjuvant ones to assist the effects of the principal component, likely with synergistic actions. In the 20 potential ingredients related to IL-6, NF-kB, FASN, and ACACA (Additional file 6: Table S6), particularly quercetin, luteolin, puerarin, apigenin, and rutin can alleviate NAFLD. Emerging evidence has revealed that quercetin is effective in reversing the symptoms of NAFLD by lowering lipid accumulation and ameliorating the lipidemic profile in vivo and in vitro [39-41]. Quercetin decreases hepatic TG content by $39 \%$, with a 1.5 -fold increase in VLDL, and upregulates spliced X-box binding protein 1 (XBP1s) expression in Male Sprague-Dawley rats [39]. Quercetin can be used as a therapeutic approach for high fat diet (HFD) induced NAFLD due to its anti-inflammatory, antioxidant and prebiotic integrative response in C57BL/6J mice [42]. Previous reports indicate that luteolin decreases the adiposity and dyslipidemia by decreasing lipogenesis and increasing fatty acid oxidation [43, 44] and anti-inflammatory [45], which contributes to protection against NAFLD. Puerarin could be a promising and practical therapeutic strategy for NAFLD by modulating PARP-1/PI3K/AKT signalling pathway [46], and by activation of AMPK and its downstream effectors involved in lipid metabolism $[47,48]$ and JAK2/ STAT3 signalling pathways in hepatocytes [48, 49]. Apigenin attenuates HFD-induced NAFLD by regulating hepatocyte lipid metabolism and oxidative stress via activation of Nrf2 [50], XO/NLRP3 pathways [51], and PI3K/ AKT signalling pathway [52]. Administration of rutin significantly curtails inflammation, fibrosis, and hepatic hyperplasia in a nitrosodiethylamine (NDEA) model of hepatocarcinogenesis in rats [53]. Rutin exhibits hepatoprotective effects in HFD-induced NAFLD by reducing hepatic lipid levels and mitigating lipid-induced oxidative injuries in male C57BL/6 mice [54].

Furthermore, 4 herbs (BC, SM, ZJ, and GU) in the formula share common ingredients (ursolic acid, quercetin, apigenin, and tanshinone iia) acting on both lipid metabolism and inflammatory-related pathways. Every herb in the formula has specific ingredients affecting key factors. The efficacy of the three additional herbs affected the improvement of MXD in NAFLD in different aspects. 


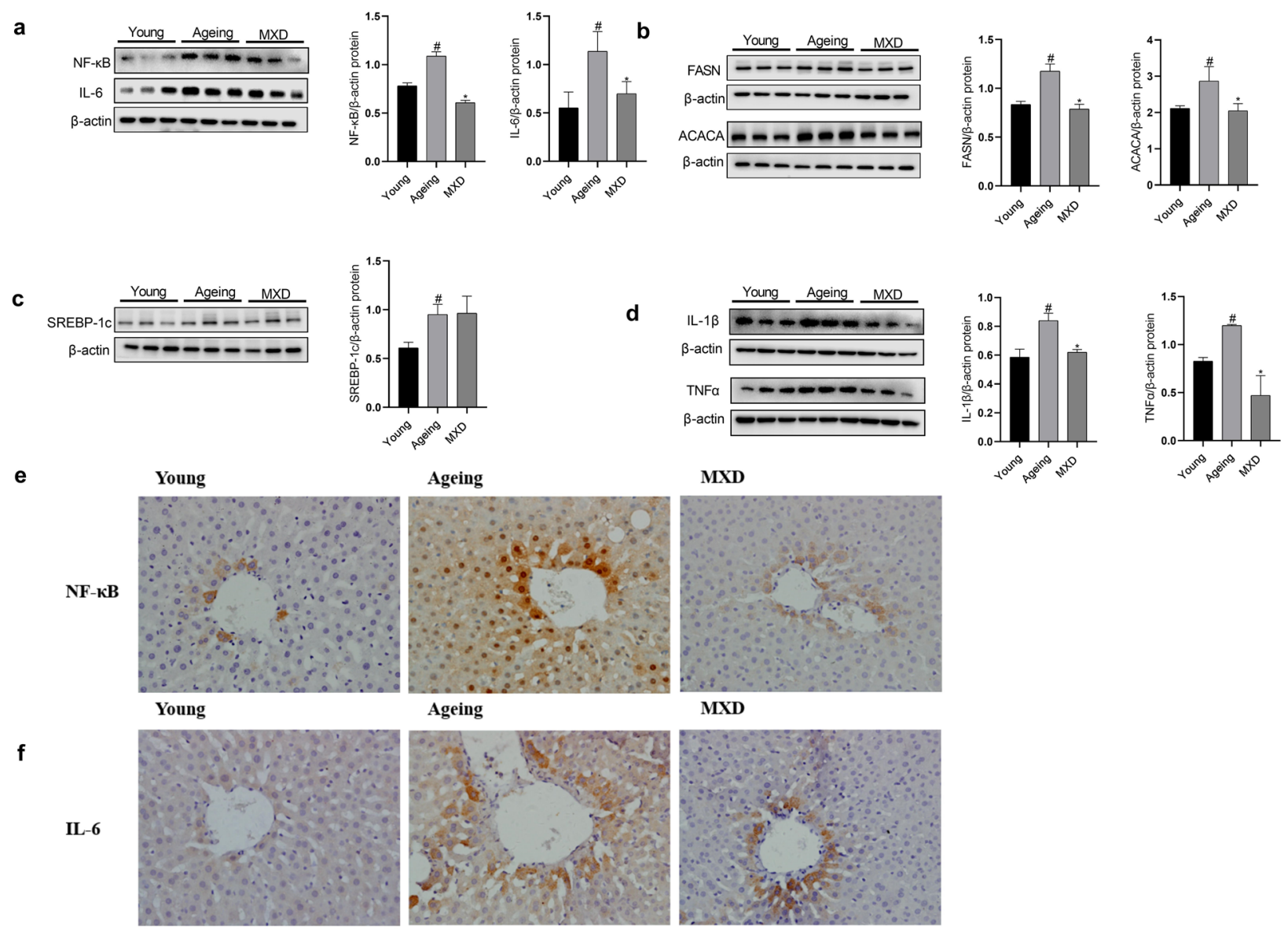

Fig. 8 Effect of MXD against NAFLD on the protein expression of inflammation and lipid metabolism-related factors in ageing female rats. a-d The protein expression of IL-6, NF-KB, FASN, ACACA, SREBP-1C, IL-1 $\beta$, and TNFa was determined by western blot. e-f The protein expression of IL-6 and NF-KB was determined by immunohistochemistry. Positive staining is brown. ${ }^{\#} \mathrm{P}<0.05$ compared to the control. ${ }^{*} \mathrm{P}<0.05$ compared to the model

Notably, in our study, SM, one of the additional herbs, ranked second only to $\mathrm{BC}$ in contributing to the modulation of lipid metabolism and inflammation, suggesting the important role of additional herbs in MXD against NAFLD. This combination probably generates a wider efficacy at a low concentration, and is apparently safer than a single drug. Therefore, it suggests that TCM offers bright prospects for the control of complex disease in a synergistic manner.

However, there are some limitations for the use of network pharmacology in our research. First, the active ingredients screened might be different than the ingredients actually absorbed in the blood of patients with NAFLD. Second, the predicted results might be impacted by possible biases to highly studied pathways/targets. In the future, we will endeavour to do more work to verify the potential mechanisms of MXD protection against NAFLD.

\section{Conclusion}

Taken together, our work generated a map detailing the effects of MXD against NAFLD by network pharmacology analysis. The work preliminarily confirmed that the inhibition of fatty acid synthesis and promotion of antiinflammatory properties underlie MXD-based protection. Based on the experimental finding, we further speculate the role of different herbs in contribution to the achievement of MXD and the results are BC and SM represents the principal herb for the treatment of ageing-related NAFLD in female rats, and other eight herbs serve as adjuvants to assist the principal component. To our knowledge, for the first time, we distinguished the auxiliary and major herbs of a formula based on network pharmacology and experimental verification, which provides a clue to the development of novel drugs and TCM modernization. As a result, our research may provide more evidence for the clinic application of MXD against NAFLD 
a

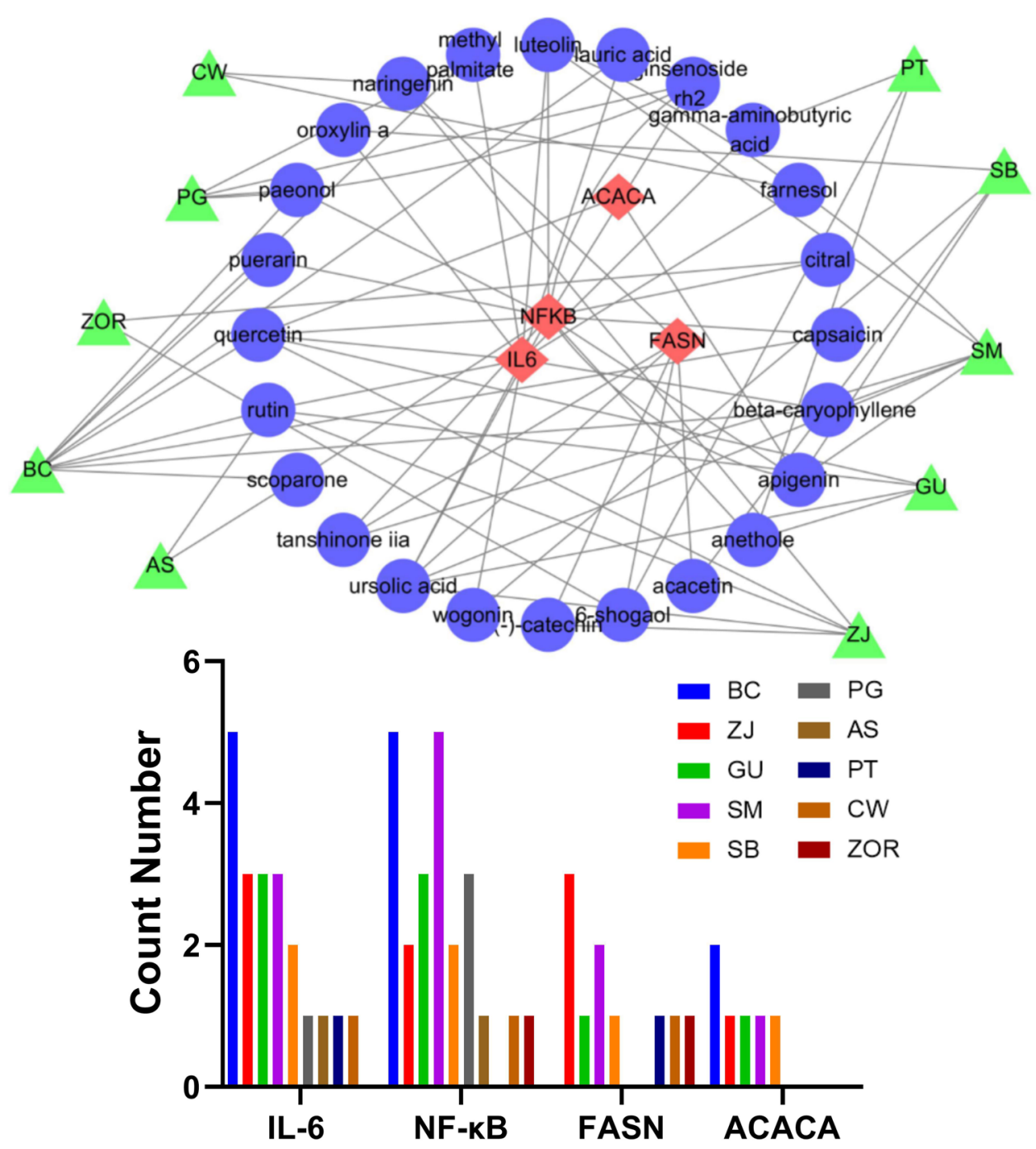

C

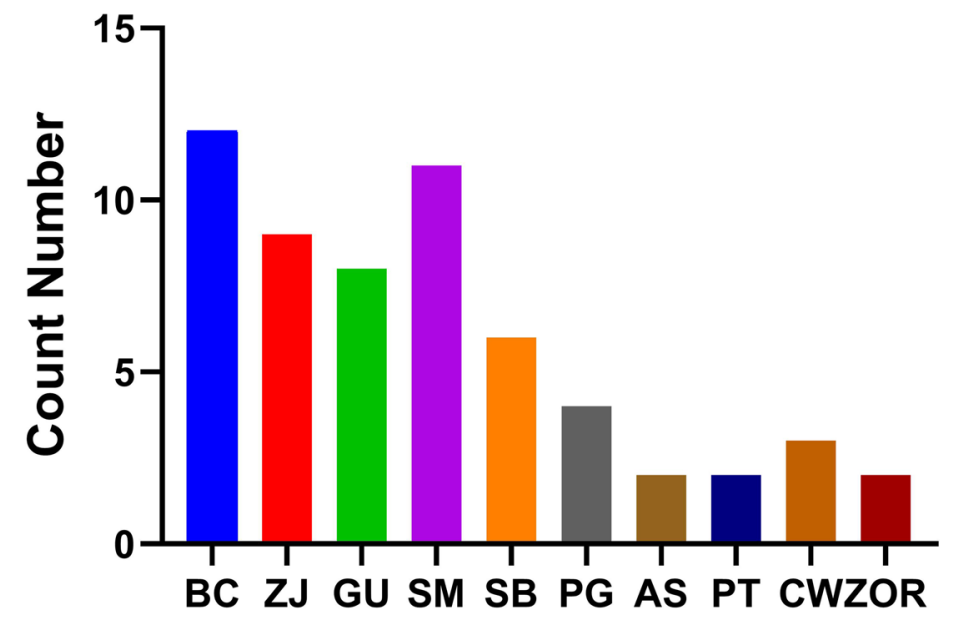

Fig. 9 The quantity of bioactive components related to verified targets (L-6, NF-KB, FASN, and ACACA) in each herb. a Construction of bioactive components of MXD related to IL-6, NF-KB, FASN, and ACACA network, containing 38 nodes and 74 edges. The blue nodes represent the biological ingredients. The green nodes represent herbs in MXD. The red nodes represent the verified targets. $\mathbf{b}$ The number of bioactive ingredients related to the corresponding verified target. c The number of bioactive ingredients related to all the verified targets 


\section{Supplementary information}

Supplementary information accompanies this paper at https://doi. org/10.1186/s13020-020-00378-y.

Additional file 1: Table S1. List of active ingredients in Modified Xiaochaihu Decoction (MXD).

Additional file 2: Table S2. List of the chemical compounds and putative targets of MXD following screening.

Additional file 3: Table S3. List of NAFLD-related targets

Additional file 4: Table S4. Results of Gene ontology (GO) functional enrichment analyses divided into three sections: Biological Process, CelIular Component, and Molecular Function.

Additional file 5: Table S5. Results of KEGG (Kyoto Encyclopedia of Genes and Genomes) pathway enrichment analysis

Additional file 6: Table S6. List of 20 potential ingredients related to IL-6, NF-KB, FASN, and ACACA.

\section{Abbreviations}

NAFLD: Non-alcoholic fatty liver disease; XCHD: Xiaochaihu Decoction; PT: Pinellia ternate (Thunb.) Breit.; PG: Panax ginseng C. A. Mey.; GU: Glycyrrhiza uralensis Fisch.; SB: Scutellaria baicalensis Georgi.; ZOR: Zingiber officinale Rose: ZJ: Ziziphus jujube Mill.; BC: Bupleurum chinense DC.; SM: Salvia miltiorrhiza Bge.; AS: Artemisia scoparia Waldst. Et Kit; CW: Curcuma wenyujin Y. H. Chen et C. Ling; TC: Total cholesterol; TG: Triglyceride; LDL: Low-density lipoprotein cholesterol; ALT: Alanine transaminase; AST: Aspartate transaminase; BMI: Body mass index; CAP: Controlled attenuation parameters; MXD: Modified Xiaochaihu Decoction; TCMSP: Traditional Chinese Medicines systems pharmacology database; PPI: Protein-protein interaction; KEGG: Kyoto Encyclopedia of Genes and Genomes; WB: Western blots; PVDF: Polyvinylidene difluoride; ALB: Serum albumin; IL-6: Interleukin-6; Casp3: Caspase-3; Egfr: Epidermal growth factor receptor; MAPK8: Mitogen-activated protein kinase 8; NF-k Nuclear factor kappa- $\beta$; SREBP-1c: Sterol regulatory element-binding protein 1 ; ChREBP: Carbohydrate response element binding protein; ACACA: Acetyl-CoA carboxylase 1; FASN: Fatty acid synthase.

\section{Acknowledgements}

I would like to thank Professor Feng Chunlai from Jiangsu University for his valuable suggestions on my article.

\section{Authors' contributions}

JW prepared academic plans for all the studies, revised the final manuscript and was responsible for the funding. SG designed this study, conducted all the experiments, searched the databases, analyzed the data, created the illustrations, and drafted the manuscript. TW and YJ participated in the study design, Western blotting, and animal administration. YL and DK participated in RT-PCR. HX and HG participated in the serum biochemical indexes, $X H$ and $Y X$ provided the precription and participated in the plan for the experiment. All authors read and approved the final manuscript.

\section{Funding}

This work was financially supported by the National Natural Science Foundation of China (Grant No: 81673659 and 81973653), the Foundation of Chongqing Health and Family Planning Commission (Grant No: ZY201702133), the Natural Science Foundation of Chongqing (municipality) (Grant No: cstc2017jcyjAX0374; cstc2018jcyjAX0176), Innovation experiment project of Chongqing Medical University (Grant No: SRIEP201918), Xinglin project of College of Traditional Chinese Medicine, Chongqing Medical University (Grant No: 201810), Project of Chongqing Yuzhong district science committee (Grant No: 20190117).

\section{Availability of data and materials}

The datasets used and/or analyzed during the current study are available from the corresponding author on reasonable request.

\section{Ethics approval and consent to participate}

All animal protocols in the study were performed in accordance with international ethical guidelines and the National Institutes of Health Guide concerning the Care and Use of Laboratory Animals. All experiments involving animals were approved by the Institutional Animal Care and Use Committee of Chongqing Medical University.

\section{Consent for publication}

Not applicable.

\section{Competing interests}

The authors declare that they have no competing interests.

\section{Author details}

${ }^{1}$ Chongqing Key Laboratory of Traditional Chinese Medicine for Prevention and Cure of Metabolic Diseases, Chongqing Medical University, Chongqing, China. ${ }^{2}$ College of Traditional Chinese Medicine, Chongqing Medical University, Chongqing, China. ${ }^{3}$ Faculty of Basic Medical Sciences, Chongqing Medical University, Chongqing, China. ${ }^{4}$ The Second Affiliated Hospital, Chongqing Medical University, Chongqing, China.

Received: 2 March 2020 Accepted: 31 Auqust 2020

Published online: 04 September 2020

\section{References}

1. Partridge L, Deelen J, Slagboom PE. Facing up to the global challenges of ageing. Nature. 2018:561:45-56. https://doi.org/10.1038/s4158 6-018-0457-8

2. Kennedy BK, Berger SL, Brunet A, Campisi J, Cuervo AM, Epel ES, et al. Geroscience: linking aging to chronic disease. Cell. 2014;159(4):9-13. https://doi.org/10.1016/j.cell.2014.10.039.

3. Gong Z, Tas E, Yakar S, Muzumdar R. Hepatic lipid metabolism and nonalcoholic fatty liver disease in aging. Mol Cell Endocrinol. 2017:455:11530. https://doi.org/10.1016/j.mce.2016.12.022.

4. Brady CW. Liver disease in menopause. World J Gastroenterol. 2015:21(25):7613-20. https://doi.org/10.3748/wjg.v21.i25.7613.

5. Banini BA, Sanyal AJ. Current and future pharmacologic treatment of nonalcoholic steatohepatitis. Curr Opin Gastroenterol. 2017;33(3):134-41. https://doi.org/10.1097/MOG.0000000000000356.

6. Chinese TJ, Medica M. In: Cui H, editor. People's Medical Publishing House Beijing: Academic; 2007. p. 2-5.

7. Takahashi Y, Soejima Y, Kumagai A, Watanabe M, Uozaki H, Fukusato T. Inhibitory effects of Japanese herbal medicines sho-saiko-to and juzen-taiho-to on nonalcoholic steatohepatitis in mice. PLoS ONE. 2014;9(1):872-9. https://doi.org/10.1371/journal.pone.0087279.

8. Chinese TJ, Medica M. In: Cui H, editor. People's Medical Publishing House. Beijing: Academic; 2007. p. 39-41.

9. Tong S. Observation on the clinical effect of Xiaochaihu decoction on nonalcoholic fatty liver disease. Asia Pac J Clin Nutr. 2017;13(11):133-4. https://doi.org/10.11954/ytctyy.201711061.

10. Li SM, Xu SJ, Li SM, Hong WX. Study on clinical experience of using xiaochaihu decoction. Chin J Integr Trad West Med. 2014;34(10):1264-6. https ://doi.org/10.7661/CJIM.2014.10.1264

11. Hu Z, Zheng X, Xue S, Hu Y. Literature research on the clinical application of xiaochaihu decoction in fatty liver disease. Chin J Integr Trad West Med. 2011;20(2):254-6. https://doi.org/10.3969/j.issn.1008-8849.2011.02.087.

12. Takahashi Y, Soejima Y, Kumagai A, Watanabe M, Uozaki H, Fukusato T. Japanese herbal medicines shosaikoto, inchinkoto, and juzentaihoto inhibit high-fat diet-induced nonalcoholic steatohepatitis in $\mathrm{db} / \mathrm{db}$ mice. Pathol Int. 2014:64(10):490-8. https://doi.org/10.1111/pin.12199.

13. Li SZ, Zhong H, Ning L, Shenghong L. Study on clinical experience of using xiaochaihu decoction. Guangming J Med. 2014;34(10):1264-6. https://doi.org/10.3969/j.issn.1003-8914.2017.16.025.

14. Qu W. Treatment of 65 cases of nonalcoholic fatty liver disease by syndrome differentiation. Henan Chin Med. 2012;32(07):52-4. https://doi. org/10.16367/j.issn.1003-5028.2012.07.056 
15. He B. Short-term curative effect observation of Modified Xiaochaihu Decoction in treating fatty liver. Zhejiang Clin Med. 2002;2002(04):276. https://doi.org/10.3969/j.issn.1008-7664.2002.04.022.

16. Yang Z, Huang X. Analysis of 48 cases of nonalcoholic fatty liver disease treated with modified Xiaochaihu Decoction. Chin J Primary Med Pharm. 2009;2009(01):159-60. https://doi.org/10.3760/cma.j.i ssn.1008-6706.2009.01.102.

17. Hong M, Li S, Wang N, Tan HY, Cheung F, Feng Y. a biomedical investigation of the hepatoprotective effect of Radix salviae miltiorrhizae and network pharmacology-based prediction of the active compounds and molecular targets. Int J Mol Sci. 2017;18(3):620. https://doi.org/10.3390/ ijms18030620.

18. Cicero A, Colletti A, Bellentani S. Nutraceutical approach to non-alcoholic fatty liver disease (NAFLD): the available clinical evidence. Nutrients. 2018;10(9):1153-70. https://doi.org/10.3390/nu10091153.

19. Wang ZQ, Zhang XH, Yu Y, Tipton RC, Raskin I, Ribnicky D, et al. Artemisia scoparia extract attenuates non-alcoholic fatty liver disease in dietinduced obesity mice by enhancing hepatic insulin and AMPK signalling independently of FGF21 pathway. Metabolism. 2013;62(9):1239-49. https ://doi.org/10.1016/j.metabol.2013.03.004

20. Kim SB, Kang OH, Lee YS, Han SH, Ahn YS, Cha SW, et al. Hepatoprotective effect and synergism of bisdemethoycurcumin against MCD diet-induced nonalcoholic fatty liver disease in mice. PLoS ONE. 2016;11(2):e0147745. https://doi.org/10.1371/journal.pone.0147745.

21. Lin AX, Chan G, Hu Y, Ouyang D, Ung C, Shi L, et al. Internationalization of traditional Chinese medicine: current international market, internationalization challenges and prospective suggestions. Chin Med. 2018;13:9-27. https://doi.org/10.1186/s13020-018-0167-z.

22. Li S, Zhang B. Traditional Chinese medicine network pharmacology: theory, methodology and application. Chin J Nat Med. 2013;11(2):110-20. https://doi.org/10.1016/S1875-5364(13)60037-0.

23. Liang $X$, Li H, Li S. A novel network pharmacology approach to analyse traditional herbal formulae: the Liu-Wei-Di-Huang pill as a case study. Mol Biosyst. 2014;10(5):1014-22. https://doi.org/10.1039/c3mb70507b.

24. Zheng J, Wu M, Wang $H$, et al. Network pharmacology to unveil the biological basis of health-strengthening herbal medicine in cancer treatment. Cancers (Basel). 2018;10(11):461. https://doi.org/10.3390/cance rs10110461.

25. Li S, Qian Y, Xie R, Li Y, Jia Z, Zhang Z, et al. Exploring the protective effect of ShengMai-Yin and Ganmaidazao decoction combination against type 2 diabetes mellitus with nonalcoholic fatty liver disease by network pharmacology and validation in KKAy mice. J Ethnopharmacol. 2019;242:11229. https://doi.org/10.1016/j.jep.2019.112029.

26. Yu G, Wang LG, Han Y, He QY. clusterProfiler: an R package for comparing biological themes among gene clusters. OMICS. 2012;16(5):284-7. https ://doi.org/10.1089/omi.2011.0118.

27. Liu L, Yao L, Wang S, et al. 6-Gingerol improves ectopic lipid accumulation, mitochondrial dysfunction, and insulin resistance in skeletal muscle of ageing rats: dual stimulation of the AMPK/PGC-1 a signalling pathway via plasma adiponectin and muscular AdipoR1. Mol Nutr Food Res. 2019:63(6):e1800649. https://doi.org/10.1002/mnfr.201800649.

28. Wang J, Ke W, Bao R, Hu X, Chen F. Beneficial effects of ginger Zingiber officinale Roscoe on obesity and metabolic syndrome: a review. Ann NY Acad Sci. 2017;1398(1):83-988. https://doi.org/10.1111/nyas.13375.

29. Buzzetti E, Pinzani M, Tsochatzis EA. The multiple-hit pathogenesis of non-alcoholic fatty liver disease (NAFLD). Metabolism. 2016;65(8):103848. https://doi.org/10.1016/j.metabol.2015.12.012.

30. Friedman SL, Neuschwander-Tetri BA, Rinella M, Sanyal AJ. Mechanisms of NAFLD development and therapeutic strategies. Nat Med. 2018;24(7):908-22. https://doi.org/10.1038/s41591-018-0104-9.

31. Tong $X, X u$ J, Lian F, et al. Structural alteration of gut microbiota during the amelioration of human type 2 diabetes with hyperlipidemia by metformin and a Traditional Chinese Herbal formula: a multicenter, randomized. Open Label Clinical Trial mBio. 2018;9(3):e02392-e2417. https:// doi.org/10.1128/mBio.02392-17.

32. Sang TT, Guo CJ, Guo DD, Wang XY. Effect of traditional Chinese medicine in inhibiting obesity and inflammatory diseases by regulating gut microbiota. Chin J Chin Mater Med. 2018;43(16):3235-42. https://doi. org/10.19540/j.cnki.cjcmm.20180423.003.
33. Liu C, Sun M, Wang L, Wang G, Chen G, Liu C, et al. Effects of Yinchenhao Tang and related decoctions on DMN-induced cirrhosis/fibrosis in rats. Chin Med. 2008:3:11-25. https://doi.org/10.1186/1749-8546-3-1.

34. Lee $G$, Jang H, Kim $Y Y$, et al. SREBP1c-PAX4 axis mediates pancreatic $\beta$-cell compensatory responses upon metabolic stress. Diabetes. 2019;68(1):8194. https://doi.org/10.2337/db18-0556.

35. Knebel B, Fahlbusch P, Dille $M$, et al. Fatty liver due to increased de novo lipogenesis: alterations in the hepatic peroxisomal proteome. Front Cell Dev Biol. 2019;7:248. https://doi.org/10.3389/fcell.2019.00248.

36. Lane EA, Choi DW, Garcia-Haro L, et al. HCF-1 regulates De Novo lipogenesis through a nutrient-sensitive complex with ChREBP. Mol Cell. 2019;75(2):357-371.e7. https://doi.org/10.1016/j.molcel.2019.05.019.

37. Horton JD, Goldstein JL, Brown MS. SREBPs: activators of the complete program of cholesterol and fatty acid synthesis in the liver. J Clin Invest. 2002;109(9):1125-31. https://doi.org/10.1172/JCl15593.

38. Ortega-Prieto P, Postic C. Carbohydrate sensing through the transcription factor ChREBP. Front Genet. 2019;10:472. https://doi.org/10.3389/fgene 2019.00472.

39. Seiva FR, Chuffa LG, Braga CP, Amorim JP, Fernandes AA. Quercetin ameliorates glucose and lipid metabolism and improves antioxidant status in postnatally monosodium glutamate-induced metabolic alterations. Food Chem Toxicol. 2012;50(10):3556-611. https://doi.org/10.1016/j. fct.2012.07.009.

40. Ulasova E, Perez J, Hill BG, Bradley WE, Garber DW, Landar A, et al. Quercetin prevents left ventricular hypertrophy in the Apo E knockout mouse. Redox Biol. 2013;1:381-6. https://doi.org/10.1016/j.redox.2013.07.001.

41. Zhu X, Xiong T, Liu P, Guo X, Xiao L, Zhou F, et al. Quercetin ameliorates HFD-induced NAFLD by promoting hepatic VLDL assembly and lipophagy via the IRE1a/XBP1s pathway. Food Chem Toxicol. 2018;114:52-60. https://doi.org/10.1016/j.fct.2018.02.019.

42. Porras D, Nistal E, Martinez-Florez S, Olcoz JL, Jover R, Jorquera F, et al. Functional interactions between gut microbiota transplantation, quercetin, and high-fat diet determine non-alcoholic fatty liver disease development in germ-free mice. Mol Nutr Food Res. 2019;63(8):180-93. https://doi.org/10.1002/mnfr.201800930.

43. Kwon EY, Kim SY, Choi MS. Luteolin-enriched artichoke leaf extract alleviates the metabolic syndrome in mice with high-fat diet-induced obesity. Nutrients. 2018;10(8):45-77. https://doi.org/10.3390/nu10080979.

44. Yin Y, Gao L, Lin H, Wu Y, Han X, Zhu Y, et al. Luteolin improves nonalcoholic fatty liver disease in $\mathrm{db} / \mathrm{db}$ mice by inhibition of liver $X$ receptor activation to down-regulate expression of sterol regulatory element binding protein 1c. Biochem Biophys Res Commun. 2017;482(4):720-6. https://doi.org/10.1016/j.bbrc.2016.11.101.

45. Abu-Elsaad N, El-Karef A. Protection against nonalcoholic steatohepatitis through targeting IL-18 and IL-1alpha by luteolin. Pharmacol Rep. 2019;71(4):688-94. https://doi.org/10.1016/j.pharep.2019.03.009.

46. Wang S, Yang FJ, Shang LC, Zhang YH, Zhou Y, Shi XL. Puerarin protects against high-fat high-sucrose diet-induced non-alcoholic fatty liver disease by modulating PARP-1/PI3K/AKT signalling pathway and facilitating mitochondrial homeostasis. Phytother Res. 2019;33(9):2347-59. https:// doi.org/10.1002/ptr.6417.

47. Xu G, Huang K, Zhou J. Hepatic AMP kinase as a potential target for treating nonalcoholic fatty liver disease: evidence from studies of natural products. Curr Med Chem. 2018;25(8):889-907. https://doi. org/10.2174/0929867324666170404142450.

48. Kang OH, Kim SB, Mun SH, Seo YS, Hwang HC, Lee YM, et al. Puerarin ameliorates hepatic steatosis by activating the PPARalpha and AMPK signalling pathways in hepatocytes. Int J Mol Med. 2015;35(3):803-9. https:// doi.org/10.3892/ijmm.2015.2074.

49. Zheng P, Ji G, Ma Z, Liu T, Xin L, Wu H, et al. Therapeutic effect of puerarin on non-alcoholic rat fatty liver by improving leptin signal transduction through JAK2/STAT3 pathways. Am J Chin Med. 2009;37(1):69-83. https:// doi.org/10.1142/S0192415X09006692.

50. Feng X, Yu W, Li X, Zhou F, Zhang W, Shen $\mathrm{Q}$ et al. Apigenin, a modulator of PPARgamma, attenuates HFD-induced NAFLD by regulating hepatocyte lipid metabolism and oxidative stress via Nrf2 activation. Biochem Pharmacol. 2017;136:136-49. https://doi.org/10.1016/j.bcp.2017.04.014.

51. Lv Y, Gao X, Luo Y, Fan W, Shen T, Ding C, et al. Apigenin ameliorates HFDinduced NAFLD through regulation of the XO/NLRP3 pathways. J Nutr Biochem. 2019;71:110-21. https://doi.org/10.1016/j.jnutbio.2019.05.015. 
52. Fan $H$, Ma X, Lin $P$, Kang Q, Zhao Z, Wang L, et al. Scutellarin prevents nonalcoholic fatty liver disease (NAFLD) and Hyperlipidemia via PI3K AKT-dependent activation of nuclear factor (Erythroid-Derived 2)-like 2 (Nrf2) in rats. Med Sci Monit. 2017;23:5599-612. https://doi.org/10.12659/ msm.907530.

53. Thomas NS, George K, Arivalagan S, Mani V, Siddique Al, Namasivayam $\mathrm{N}$. The in vivo antineoplastic and therapeutic efficacy of troxerutin on rat preneoplastic liver: biochemical, histological and cellular aspects. Eur J Nutr. 2017;56(7):2353-66. https://doi.org/10.1007/s00394-016-1275-0.

54. Liu Q, Pan R, Ding L, Zhang F, Hu L, Ding B, et al. Rutin exhibits hepatoprotective effects in a mouse model of non-alcoholic fatty liver disease by reducing hepatic lipid levels and mitigating lipid-induced oxidative injuries. Int Immunopharmacol. 2017;49:132-41. https://doi.org/10.1016/j. intimp.2017.05.026.

\section{Publisher's Note}

Springer Nature remains neutral with regard to jurisdictional claims in published maps and institutional affiliations.
Ready to submit your research? Choose BMC and benefit from:

- fast, convenient online submission

- thorough peer review by experienced researchers in your field

- rapid publication on acceptance

- support for research data, including large and complex data types

- gold Open Access which fosters wider collaboration and increased citations

- maximum visibility for your research: over 100M website views per year

At BMC, research is always in progress.

Learn more biomedcentral.com/submissions 